\title{
Estudio Descriptivo de los Accesos Quirúrgicos Utilizados en el Tratamiento de Fracturas del Complejo Zigomático Orbitario
}

\author{
Descriptive Study of Surgical Approach for Zygomatic Complex Fractures \\ *,** Sergio Olate ; "Sergio Monteiro Lima Junior; "Renato Sawazaki; "Roger William Fernandes Moreira \& "Marcio de Moraes
}

OLATE, S.; LIMA JUNIOR, S. M.; SAWAZAKI, R.; MOREIRA, R. W. F. \& DE MORAES, M. Estudio descriptivo de los accesos quirúrgicos utilizados en el tratamiento de fracturas de complejo zigomático orbitario. Resultados preliminares. Int. J. Morphol., 27(2):299304, 2009.

RESUMEN: El objetivo del presente estudio fue evaluar los accesos quirúrgicos utilizados para el abordaje del complejo zigomático orbitario (CZO) y arco zigomático (AZ). Fue diseñado un estudio de tipo retrospectivo, evaluando las fichas clínicas de pacientes atendidos entre el 1 de Abril del año 1999 y el 31 de Diciembre del año 2008. Fueron estudiadas variables sociodemográficas y características de la fractura, tales como presencia de más de una fractura facial y desplazamiento del fragmento óseo. Se realizo un estudio descriptivo de los diferentes accesos quirúrgicos utilizados y cuando fue necesario se estudió la asociación estadística con la prueba chi-cuadrado para variables nominales, estableciendo significancia si $\mathrm{p}<0,05$. Ciento cincuenta y tres pacientes fueron sometidos a tratamiento quirúrgico de fractura de CZO con 251 accesos quirúrgicos. El acceso más efectuado fue el intrabucal, seguido del acceso subciliar y supraciliar. Siempre existió mayor utilización de accesos para pilar zigomaticomaxilar, seguidos por accesos para reborde infraorbitario y sutura frontozigomática, con pocas diferencias entre ellos. No fue posible encontrar asociación estadística entre las variables estudiadas y la cantidad de accesos para el tratamiento quirúrgico de fracturas de CZO. Los accesos quirúrgicos deben responder a las necesidades individuales de cada caso, intentando obtener indicaciones precisas, más que preferencias individuales de cada cirujano.

\section{PALABRAS CLAVE: Trauma maxilofacial; Fractura de zigoma; Accesos quirúrgicos.}

\section{INTRODUCCIÓN}

El tratamiento de las fracturas maxilofaciales ha evolucionado con el desarrollo de técnicas que permitan la corrección anatómica y funcional de las estructuras óseas. De esta forma, el tratamiento quirúrgico y no quirúrgico han sufrido modificaciones importantes en cuanto a su indicación en traumatología maxilofacial.

La epidemiología de las fracturas maxilofaciales presenta constantes variaciones debido a cambios en la forma de vida de las personas y a la composición poblacional en determinado momento (Ogundare et al., 2003; Medina et $a l ., 2006)$; por lo que la etiología del trauma maxilofacial es cada vez más cercano a traumas de alto impacto, asociados, en buena parte, con accidentes automovilísticos. Sin embargo, el caso de las fracturas de CZO y AZ presenta algunas variaciones, donde una investigación previa de nuestro servicio evidenció una alta asociación con accidentes de bicicleta y agresiones físicas, demostrando una tendencia a trau- mas de baja a media intensidad (Gomes et al., 2006). En consideración a estas variaciones, podemos decir que la prevalencia de fracturas de $\mathrm{CZO}$, oscila generalmente entre el 20\% y el 30\% (Chrcanovic et al., 2004; Gassner et al., 2003).

El padrón frecuente de fractura de CZO es representado por la desarticulación del zigoma en todos sus puntos de articulación realizando un movimiento hacia posterior, inferior y lateral, determinando perdida de proyección anteroposterior y, en algunos casos, alteración en el ancho del tercio medio facial, permitiendo así demandas estéticas de los pacientes (Zingg et al., 1992). Esta situación se asocia características clásicas de las fracturas de CZO como: 1) modificación de la función visual (diplopía) por alteraciones en la función del nervio oculomotor o encarcelamiento muscular, 2) fractura de arco zigomático que bloquea el movimiento del proceso coronoides, dificultando la abertura bucal, 3) anestesia o parestesia regional por lesión del 
nervio infraorbitario, parte del primer brazo del nervio trigémino (Bailey \& Goldwasser, 2004).

Cambios terapéuticos dirigidos hacia el uso de sistemas de fijación interna rígida (FIR) establecieron nuevos padrones de resultado en el tratamiento quirúrgico, siendo mejor resueltas las necesidades estéticas asociadas a estas fracturas (Ellis, 1997); junto a ello, la visualización de reducción y estabilización de la fractura determinó la necesidad de realizar diferentes accesos quirúrgicos que permitieran realizar un procedimiento con resultados funcionales y estéticos satisfactorios (Becelli et al., 2002).

Aún es controversial la elección del mejor tratamiento quirúrgico en términos de accesos quirúrgicos y FIR. Parte de esta controversia se puede observar con las directrices de tratamiento en diferentes centros de traumatología oral y maxilofacial; así Markowitz \& Manson (1998) preconizan la visualización de tres puntos y uso de FIR en dos para tener seguridad de la alineación anatómica y estabilidad postquirúrgica. Ellis \& Kittidumkerng (1996) proponen un algoritmo que evalúa reducción y estabilidad secuencial, iniciándose por el acceso intrabucal. Lee et al. (2006) sugieren un acceso transconjuntival para reborde infraorbitario y sutura frontozigomática para realizar reducción y fijación de tan solo esos dos puntos. Kovacs \& Ghahremani (2001) sugieren, por otra parte, el acceso supraciliar para reducción y fijación junto a reducción de cuerpo de zigoma con gancho de acceso extrabucal.

El objetivo de esta investigación fue identificar los accesos quirúrgicos utilizados en nuestro departamento quirúrgico y describir algunas de sus ventajas y desventajas.

\section{MATERIAL Y MÉTODO}

Se diseñó un estudio longitudinal retrospectivo para evaluar las fichas clínicas de pacientes que consultaron por trauma maxilofacial entre el 1 de Abril del año 1999 y el 31 de Diciembre del año 2008 en el Servicio de Cirugía y Traumatología Oral y Maxilofacial de la Facultad de Odontología de Piracicaba de la Universidad Estadual de Campinas (FOP-UNICAMP). Fueron excluidos los documentos que estaban incompletos o que presentaban deficiencias en sus descripciones.

De esta forma, la muestra incluyó todos los pacientes sometidos a tratamiento quirúrgico por fractura de $\mathrm{CZO}$ o AZ aislada o no y quienes completaron una forma de consentimiento informado donde autorizaron la utilización de la información en la presente investigación.
Las evaluaciones recolectadas incluyeron la situación sociodemográfica de los pacientes, tales como sexo, edad y etiología del trauma. El trauma de CZO y AZ fue diagnosticado como unilateral, bilateral, aislada o compuesta con otras fracturas faciales y finalmente si se presentaba conminuta o con trazos únicos. El diagnóstico fue realizado por nuestro Servicio en 8 hospitales correspondientes al área geográfica de las ciudades de Piracicaba, Limeira y Rio Claro (São Paulo, Brasil) con métodos de evaluación clínica y métodos imagenológicos que incluían proyecciones occipitomentonianas en $10^{\circ}, 15^{\circ}$ y/o $30^{\circ}$, proyección submentovertex, proyección facial posteroanterior y anteroposterior y finalmente tomografía computarizada (con imagen axial y coronal) para la planificación quirúrgica.

Fueron analizadas también, las características del procedimiento quirúrgico, incluyendo los tipos de accesos quirúrgicos utilizados en el abordaje de los pacientes, asociando variables como desplazamiento del fragmento óseo y la presencia de otras fracturas maxilofaciales.

Los datos fueron recolectados mediante tablas realizadas en el software Microsoft Office Excel 2007 (Microsoft Corporation®), efectuándose un análisis descriptivo para cada variable, para lo cual se utilizó el software estadístico BioStat 5.0® para el análisis mediante la prueba estadística de chi-cuadrado con diferencia estadística establecida cuando $\mathrm{p}<0,05$.

\section{RESULTADOS}

Los presentes datos son parte de una evaluación retrospectiva de 10 años de trauma maxilofacial atendido por el Servicio de Cirugía y Traumatología Oral y Maxilofacial de la FOP-UNICAMP. Sobre un universo de 2787 pacientes, con 532 casos de fractura de CZO y/o AZ siendo finalmente 154 pacientes sometidos a tratamiento quirúrgico.

La muestra fue integrada principalmente por pacientes de sexo masculino $(82,35 \%)$, superando ampliamente a los pacientes de sexo femenino. La edad media de la muestra fue de 31,6 años y cerca del $90 \%$ correspondió a pacientes económicamente activos.

El tipo de impacto relacionada al trauma de CZO fue de baja a media intensidad en poco más que la mitad de los casos $(56,2 \%)$, correspondiendo a accidentes deportivos, caídas, accidentes laborales, accidentes ciclísticos. Traumas de gran impacto como accidentes vehiculares, de motocicleta y atropellamiento correspondieron al 43,8\% de los casos. 
Doscientos cincuenta y un accesos quirúrgicos fueron realizados para el abordaje de estos pacientes, representando un promedio de 1,64 accesos por paciente. La Fig. 1 muestra la distribución según la cantidad y tipo de accesos realizados en los pacientes.

El 55,5\% de los pacientes fue sometido a intervención con solo un acceso quirúrgico; $24,8 \%$ de los pacientes presentó 2 accesos quirúrgicos, mientras que $19,7 \%$ de los pacientes fue intervenido con 3 accesos quirúrgicos (Fig. 2). En relación al número de fracturas faciales asociadas a fracturas de CZO,

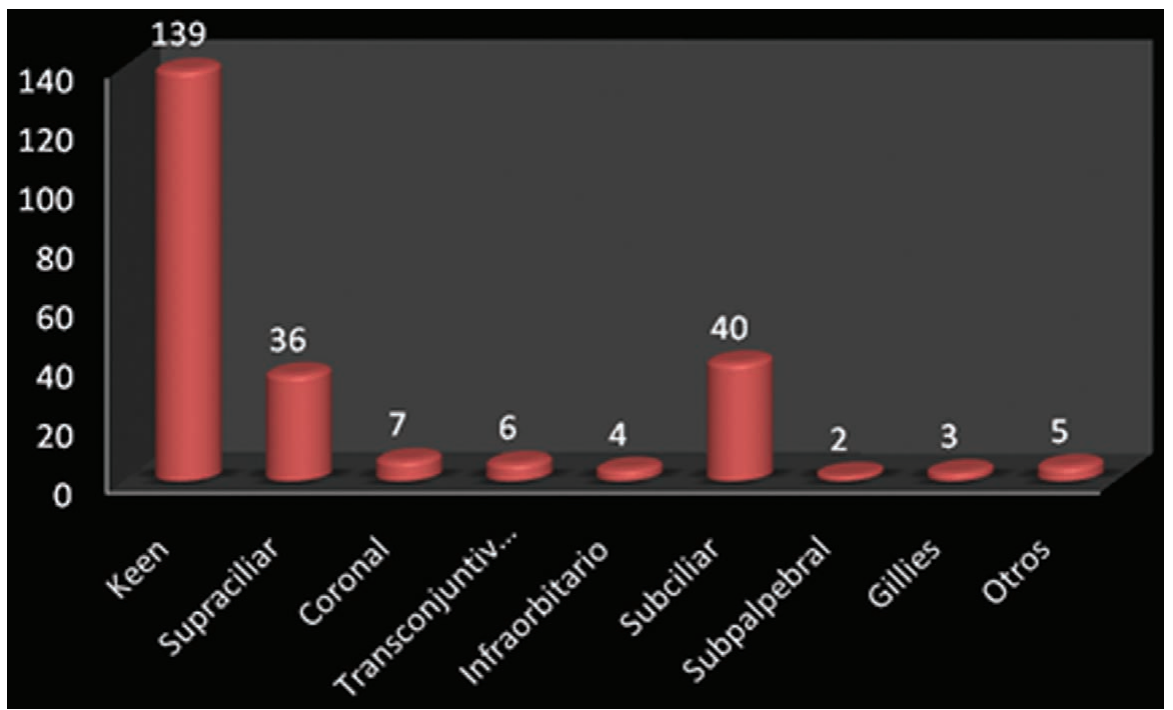

Fig. 1. Distribución de pacientes según los accesos quirúrgicos utilizados en el tratamiento de fractura de CZO.

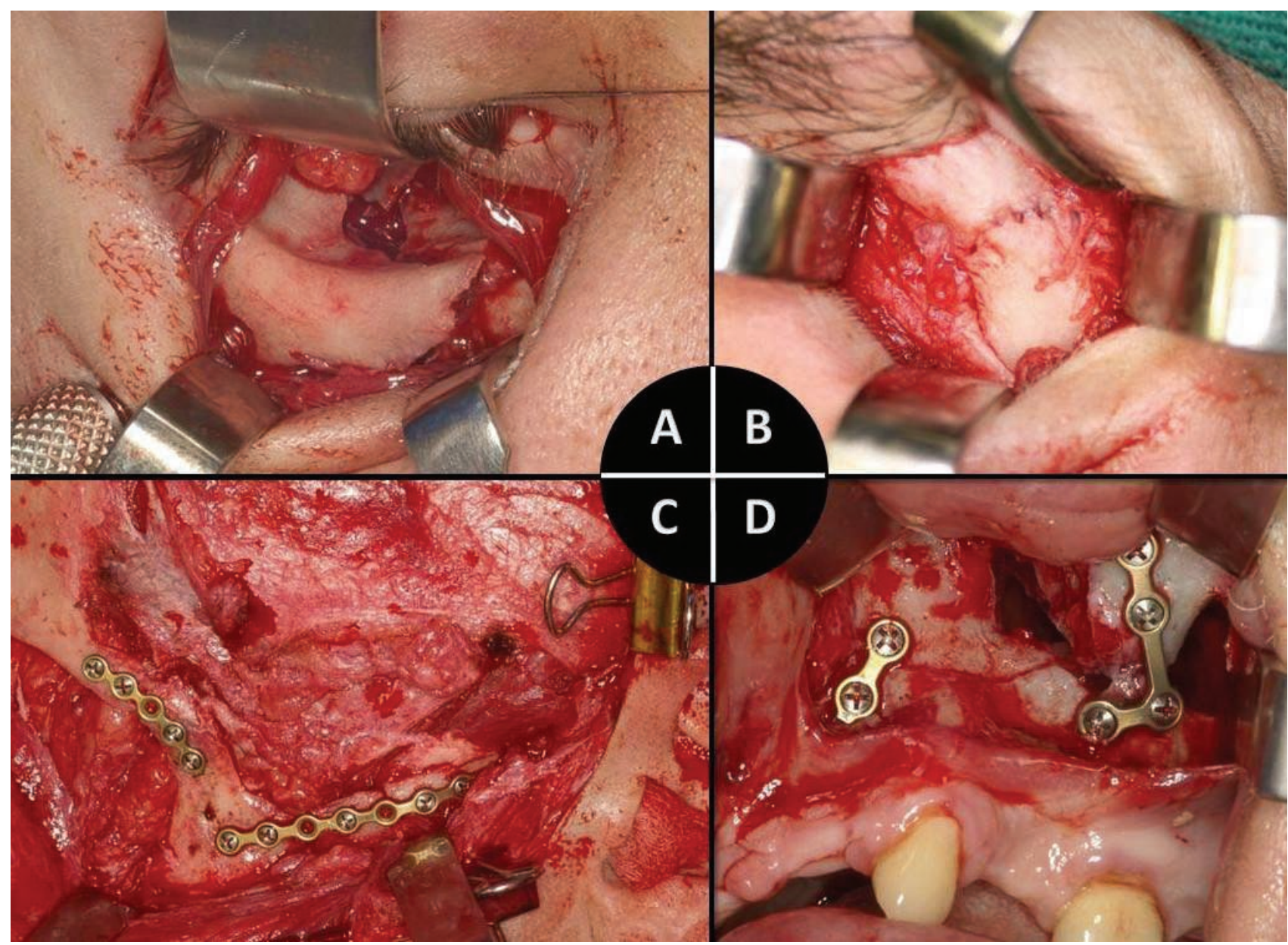

Fig. 2. Accesos quirúrgicos más utilizados en el tratamiento de fracturas de CZO. A. Acceso subciliar para exploración de piso de orbita; B. Acceso palpebral superior para exposición de sutura frontozigomática; C. Acceso coronal con extensión preauricular para reducción y fijación interna rígida de fractura en región de sutura frontozigomática y arco zigomático. D. Acceso intraoral para exposición de pilar zigomaticomaxilar y fijación del mismo con placas y tornillos del sistema 2.0. 
en 36 casos se constató la presencia de 2 grupos óseos; en 18 casos fractura de 3 grupos óseos y en solo un caso más de 3 segmentos faciales. El $62,7 \%$ de los casos correspondió a fracturas aisladas de $\mathrm{CZ}$ y/o AZ.

Después de aplicar el test de chi-cuadrado para muestras con variables nominales, no fue posible observar asociación estadísticamente significativa entre el grado de desplazamiento del fragmento del CZO y la cantidad de accesos quirúrgicos para su tratamiento y tampoco entre la cantidad de fracturas faciales y la cantidad de accesos quirúrgicos (Tabla I).

Tabla I. Evaluación estadística de accesos quirúrgicos según la cantidad de fracturas y el desplazamiento óseo de la fractura de CZO.

\begin{tabular}{lll}
\hline Accesos & Variable & $p$ \\
\hline \multirow{3}{*}{ 1 acceso } & 1 fractura & 0,8829 \\
& 2 fracturas & 0,2668 \\
& 3 o más fracturas & 0,3352 \\
& Desplazamiento & 0,1401 \\
\hline \multirow{2}{*}{ accesos } & 1 fractura & 0,9502 \\
& 2 fracturas & 0,8457 \\
& 3 o más fracturas & 0,9011 \\
& Desplazamiento & 0,5762 \\
\hline \multirow{3}{*}{3 accesos } & 1 fractura & 0,9129 \\
& 2 fracturas & 0,308 \\
& 3 o más fracturas & 0,2595 \\
& Desplazamiento & 0,2685 \\
\hline
\end{tabular}

\section{DISCUSIÓN}

El manejo del trauma maxilofacial es un constante desafío para cirujanos maxilofaciales debido al gran compromiso funcional y estético de áreas asociadas. Históricamente, la recuperación de la función fue el gran objetivo del manejo quirúrgico del trauma; sin embargo, actualmente la recuperación estética inmediata es una necesidad y una búsqueda frecuente en nuestros pacientes (Ellis).

Nuestros resultados revelan que la cantidad de accesos quirúrgicos no presenta relación con la complejidad de la fractura en términos del grado de desplazamiento del CZO o en relación con la cantidad de fracturas del componente maxilofacial. Por estos motivos creemos que la asociación de nuevas variables puede ser estudiada para identificar alguna relación significativa. El tipo de acceso tampoco presento relaciones significativas con las variables estudiadas, determi- nando con ello que la elección del cirujano, basada en otras circunstancias inicialmente no incluidas en esta investigación, presenta un componente fundamental en el momento de escoger los accesos quirúrgicos (Ellis \& Kittidumkerng). Técnicamente, cuando es necesario un abordaje quirúrgico total para $\mathrm{CZO}$, sería necesario realizar hasta 3 accesos quirúrgicos, siendo las opciones quirúrgicas limitadas y efectivas.

El acceso quirúrgico más utilizado fue el de tipo intraoral, indicado para acceder al pilar zigomáticomaxilar. Ellis \& Kittidumkerng propusieron este punto como el área anatómica inicial para comenzar el tratamiento quirúrgico de fractura de CZO aislado. Este acceso se caracteriza por ser relativamente simple, estético y de fácil sutura; según Markowitz \& Manson este sitio es un buen punto de referencia para una reducción adecuada de la fractura. Nuestros datos muestran que la gran mayoría de nuestros pacientes presentaron este abordaje, coincidiendo con los resultados de Ellis \& Kittidumkerng (Fig. 1).

Los accesos para reborde infraorbitario son variados y su indicación debería obedecer a las regiones anatómicas próximas para acceder. El acceso subciliar fue el más efectuado en nuestra muestra $(26,14 \%)$; si bien presentaría, teóricamente, mayores complicaciones que el acceso transconjuntival (3,92\%), en términos de formación de ectropión y entropión, cuando son respetadas las técnicas de incisión, disección y sutura, la tendencia es a la homogenización de los resultados con el acceso transconjuntival (Waite \& Carr, 1991; Wilson \& Ellis, 2006). Una situación importante es la referida a las posibles complicaciones de conjuntiva palpebral, que cuando se presentan, deben ser manejadas por un profesional con entrenamiento especial en manejo de conjuntiva (Wilson \& Ellis; Kushner, 2001). Complicaciones en piel, como las producidas por el acceso subciliar o incluso subtarsal, podrían ser manejadas con más facilidad por cirujanos sin entrenamiento especial (Rohrich et al., 2003). Los accesos en piel también ofrecen una adecuada aproximación a la región naso-orbitoetmoidal (NOE) (Holtmann et al., 1981), lo que es difícil de obtener con el acceso transconjuntival, debiendo realizar maniobras quirúrgicas de mayor detalle y complejidad para alcanzar esta estructura anatómica (Edgin et al., 2007). Por otra parte Lee et al., relataron la efectividad del acceso transconjuntival con cantotomía lateral para acceder al reborde infraorbitario y sutura frontozigomática en 40 pacientes tratados con placas absorbibles y con placas de titanio. En nuestro servicio, los accesos quirúrgicos son planificados según las regiones anatómicas a abordar y según la complejidad de la fractura. Creemos que esta situación obedece a la mejor visualización y a la capacidad de realizar una correcta técnica de FIR.

Otro punto importante está en relatos de algunos investigadores que señalan que el acceso subciliar presentaría 
más complicaciones que los obtenidos con el acceso subtarsal (Rohrich et al.; Bagain et al., 2008); sin embargo, en nuestra opinión las ventajas estéticas que ofrece el acceso subciliar son importantes al ser comparadas con otros accesos en piel, lo que llevaría a su indicación. Siendo el acceso subtarsal rápido y efectivo (Holtmann et al.), el acceso subciliar ofrecería la misma exposición de tejidos, con un mayor tiempo de ejecución pero con resultados estéticos más satisfactorios. Otro acceso utilizado por nuestro equipo quirúrgico fue el infraorbitario; este acceso se utilizo solamente en el año 1999 con 4 casos y se descartó de nuestro trabajo diario debido a las mayores complicaciones estéticas que presenta.

El manejo de la sutura frontozigomática es un punto importante principalmente en la fijación de la fractura, más que en la evaluación de la alineación de la fractura de CZO (Markowitz \& Manson). Fueron utilizados el acceso supraciliar en el $23,5 \%$ de los casos y el acceso coronal en el $4,57 \%$ de los casos. Nuestros resultados muestran que el acceso palpebral superior fue utilizado en sólo un caso debido a la posibilidad de complicaciones anatómicas con el acceso superciliar (lesión a la glándula lacrimal). De los 52 pacientes presentados por Kovacs \& Ghahremani, el 84,6\% fue sometido a un acceso supraciliar con FIR; solo el 7,68\% requirió la exposición y fijación de otro punto anatómico del CZO, señalando que solo fracturas conminutas justificarían la exposición y fijación de más de un punto. Según esos autores, la sutura frontozigomática sería un buen punto de reducción anatómica, estabilización y fijación de la fractura. Por otra parte, fue establecido que el mejor punto de evaluación de la alineación es la sutura esfenozigomática, inspeccionada también a través de accesos para sutura frontozigomática
(Markowitz \& Manson). En nuestra experiencia, el acceso supraciliar es caracterizado por ser de rápida ejecución y con conservación estética toda vez que es realizado en región de cejas, en el arco supraciliar (Ellis \& Zide, 2006)

Cuando existe fractura de arco zigomático u otras fracturas faciales asociadas como región de hueso frontal o región de complejo naso orbito etmoidal, el acceso coronal presenta grandes ventajas. Entre ellas destaca la completa exposición del área que permite una reducción y estabilidad anatómica (He et al., 2007). La extensión pre-auricular es utilizada en la reducción y fijación de fracturas de arco zigomático, lo que permite devolver la proyección anteroposterior y finalmente el ancho facial con la reposición del CZO y fijación del AZ. De los casos estudiados, sólo 7 pacientes recibieron abordaje con acceso coronal; fracturas de arco zigomático aislado pueden ser tratadas mediante acceso de guillis o acceso de keen. Guillis estuvo presente en 3 casos quirúrgicos, mientras que el acceso de keen fue objetivo de reducción de arco en 2 casos. Excepcionalmente, fue utilizado en un caso el acceso de Al Kayat para reducción de AZ junto al abordaje de ramo mandibular homolateral. Otro caso que presento sólo fractura de arco zigomático y NOE fue abordado con acceso coronal.

Finalmente, la proporción de accesos quirúrgicos utilizados responde a las variables y al algoritmo propuesto por Ellis. La evaluación de la reducción de las fracturas complejas de CZO debe ser confirmada por tantos accesos como sea necesario, evaluando correctamente la reducción anatómica. Así los accesos quirúrgicos deberían responder a las necesidades individuales de cada caso más que a las preferencias del cirujano.

OLATE, S.; LIMA JUNIOR, S. M.; SAWAZAKI, R.; MOREIRA, R. W. F. \& DE MORAES, M. Descriptive study of surgical approach for zygomatic complex fractures. Preliminary results. Int. J. Morphol., 27(2):299-304, 2009.

SUMMARY: The aim of this research was to evaluated the surgical approach for zygomatic complex and zygomatic arch fracture. Was doing a retrospective study, evaluating clinical charts of patients with treatment between April 1 of 1999 and December 31, 2008. Were study sociodemographic variables, type and quantitative fracture and displacement of osseous fragment. A descriptive analysis was do it surgical approach used and when was necessary, realized a statistical analysis with Chi-Square test for nominal variables, with $\mathrm{p}<0.05$ for significant statistic. One hundred fifty tree patient was surgically treated for ZC fracture, doing 251 surgical approach. More realized approach was intraoral, follow for subciliary and superciliary approach. Always exist more approach for zygomatimaxillary pillar, follow to infraorbitary rim and frontozygomatic suture, with a little difference. Was not possible show statistic association between de veriables and the quantity of surgical approach for ZC fractures. Surgical approach was response to individual necessity, obtained certain indications more than surgeon preferences.

KEY WORDS: Maxillofacial trauma; Zigoma fracture; Surgical approach.

\section{REFERENCIAS BIBLIOGRÁFICAS}

Bagain, Z. H.; Malkawi, Z.; Hadidi, A. \& Rajab, L. D. Subtarsal approach for orbital floor repair: a long-term follow-up of 12 cases in a Jordanian teaching hospital. J. Oral Maxillofac. Surg., 66:45-50, 2008.
Bailey, J. \& Goldwasser, M. Management of zygomatic complex fracture. In: Miloro, M.; Ghali, G. E.; Larsen, P. E. \& Waite, P. D. (Ed). Peterson's principles of oral and maxillofacial surgery. Hamilton, Canada, BC Decker 
Inc, 2004. pp.445-62.

Becelli, R.; Carboni, A.; Cerulli, G.; Perugini, M. \& Lannetti, G. Delayed and inadequately treated malar fractures: evolution in the treatment, presentation of 77 cases, and review of the literature. Aesth. Plast. Surg., 26:134-8, 2002.

Chrcanovic, B. R.; Freire-Maia, B.; Souza, N. L.; Araujo, V. O. \& Abreu, M. H. N. G. Facial fractures: a 1-year retrospective study in a hospital in Belo Horizonte. Braz. Oral Res., 18:322-8, 2004.

Edgin, W. A.; Morgan-Marshall, A. \& Fitzsimmons, T. D. Transcaruncular approach to medial orbital wall fractures. J. Oral Maxillofac. Surg., 11:2345-9, 2007.

Ellis, E. 3rd. \& Kittidumkerng, W. Analysis of treatment for isolated zygomaticomaxillary complex fractures. J. Oral Maxillofac. Surg., 54:386-400, 1996.

Ellis, E. 3rd. Fractures of the zygomatic complex and arch. In: Fonseca, R.; Walker, R.; Betts, N. \& Dexter-Barber, H. (Ed): Oral and Maxillofacial Trauma. Philadelphia, WB Saunders Company, 1997. pp.571-652.

Ellis, E. 3rd. \& Zide, M. Acesso superciliar supra-orbital. In: Acessos cirúrgicos ao esqueleto facial. São Paulo, Livraria Santos Editora, 2006. pp.63-7.

Gassner, R.; Tuli, T.; Hächl, O.; Rudisch, A. \& Ulmer, H. Cranio-maxillofacial trauma: a 10 year review of 9543 cases with 21067 injuries. J. Craniomaxillofac. Surg., 31:51-61, 2003.

Gomes, P. P.; Passeri, L. A. \& Albergaria-Barbosa, J. R. A 5year retrospective study of zygomatico-orbital complex and zygomatic arch fractures in São Paulo State, Brazil. J. Oral Maxillofac. Surg., 64:63-7, 2006.

He, D.; Zhang, Y. \& Ellis, E. 3rd. Panfacial fractures: analysis of 33 cases treated late. J. Oral Maxillofac. Surg., 65:2459-65, 2007.

Holtmann, B.; Wray, R.C. \& Little, A. G. A randomized comparison of four incisions for orbital fractures. Plastic. Reconstr. Surg., 67:731-7, 1981.

Kovacs, A. F. \& Ghahremani, M. Minimization of zygomatic complex fracture treatment. Int. J. Oral Maxillofac. Surg., 30:380-3, 2001.

Kushner, G. Surgical approach to the infraorbital rim and orbital floor: the case for the transconjunctival approach J. Oral Maxillofac. Surg., 64:108-10, 2006.

Lee, P. K.; Lee, J. H.; Choi, Y. S.; Oh, D. Y; Rhie, J. W.; Han, K. T. \& Ahn, S. T. Single transconjuntival incision and two-point fixation for the treatment of noncomminuted zygomatic complex fracture. J. Korean Med. Sci., 21:1080-5, 2006.

Markowitz, B. \& Manson, P. N. Zygomatic complex fracture. In Prein, J. (Ed): Manual of internal fixation in the cranio-facial skeleton: techniques recommended by the A.O./A.S.I.F. Maxillofacial group. New York: SpringerVerlag, 1998. pp.133-8.

Medina, M. J.; Molina, P.; Bobadilla, L.; Zaror, R. \& Olate, $\mathrm{S}$. Fracturas maxilofaciales en individuos chilenos. Int. J. Morphol., 24(3):423-8, 2006.

Ogundare, B.; Bonnick, A. \& Bayley, N. Pattern of mandibular fractures in a urban major trauma center. $J$. Oral Maxillofac. Surg., 61:713-8, 2003.

Rohrich, R.; Janis, J. \& Adams, W. Subciliary versus subtarsal approaches to orbitozygomatic fractures. Plastic. Reconstr. Surg., 111:1708-13, 2003.

Waite, P. D. \& Carr, D. D. The transconjunctival approach for treating orbital trauma. J. Oral Maxillofac. Surg., 49:499-503, 1991.

Wilson, S. \& Ellis, E. 3rd. Surgical approach to the infraorbital rim and orbital floor: the case for the subtarsal approach. J. Oral Maxillofac. Surg., 64:104-7, 2006.

Zingg, M.; Laedrach, K.; Chen, J.; Chowdhury, K.; Vullemin, T.; Sutter, F. \& Raveh, J. Classification and treatment of zygomatic fractures: a review of 1,025 cases. J. Oral Maxillofac. Surg., 50:778-90, 1992.

\section{Dirección para correspondencia: \\ Dr. Marcio de Moraes \\ Av. Limeira 901 \\ Caixa Postal 52 \\ CEP 13414-903 \\ Piracicaba - SP \\ BRASIL}

\section{E mail: solate@fop.unicamp.br} mmoraes@fop.unicamp.br 